\title{
AMPPS_CNC IN AN AUTOMATED MODULAR PROCESS PLANNING SYSTEM FOR ROTATIONAL PART
}

\author{
Shinde B. $\mathrm{M}^{1}$ and Dr. Khodke P.M ${ }^{2}$, \\ ${ }^{1}$ Department of Mechanical Engineering DCOER, Pune-41 \\ bharat_mshindeeyahoo.co.in \\ ${ }^{2}$ Principal GCOE Karad \\ pmkhodke@yahoo.com
}

\begin{abstract}
The decision on sequencing of operations, tool travel and machining time calculations is done in microplanning. After a set of processes has been selected for all the features of part, the sequencing task begins by searching and analysing relationship between features. This is done by rule to detect geometrical interactions and the appropriate precedence relationship in knowledge base. For deciding sequence of operations precedence relationship among the different operations is developed using decision rules.
\end{abstract}

CNC program is generated based on the process plan of a given component. The generated CNC program is then validated by simulation software before giving it to CNC machine.

\section{KEYWORDS}

Micro planning, Automated modular process planning, Generative process planning, Computer aided design,

\section{INTRODUCTION}

Micro planning deals with decision on sequencing of operations, calculations of tool travel and machining time. As a part may contain many features, a proper sequence for machining these features is vital in achieving efficient and high quality manufacturing of the part. Operation sequencing deals with the problem of determining in what order to perform a set of selected operations such that the resulting sequence satisfies the precedence constraints established by both parts and operation. The objective of process sequencing is to minimise the total time to produce a part.

CNC machine tools require a set of commands in order to carry out desired machining operation. The job of creating this set of commands is known as programming. Programming is the process of coding machining conditions such as machining dimensions with reference points. These dimensions are derived by correctly allocating the $\mathrm{X}$ and $\mathrm{Z}$ axis. The machine tool may have facility of both manual part programming as well as programming using $\mathrm{CNC}$ programming languages such as APT, ADAPT, COMPAPT etc. 


\section{Micro Planning ANd CNC Programming System}

A modular program is developed for each of the function in Visual Basic having connectivity between them. Visual Basic has been selected as a programming language as it has compatibility with Auto-CAD and it is more users friendly. An output of each step is taken as an input for subsequent step. A structure as shown in fig.1 facilitates accurate transfer of data corresponding to each feature from one step to other step. Each of the step is described in subsequent sections.

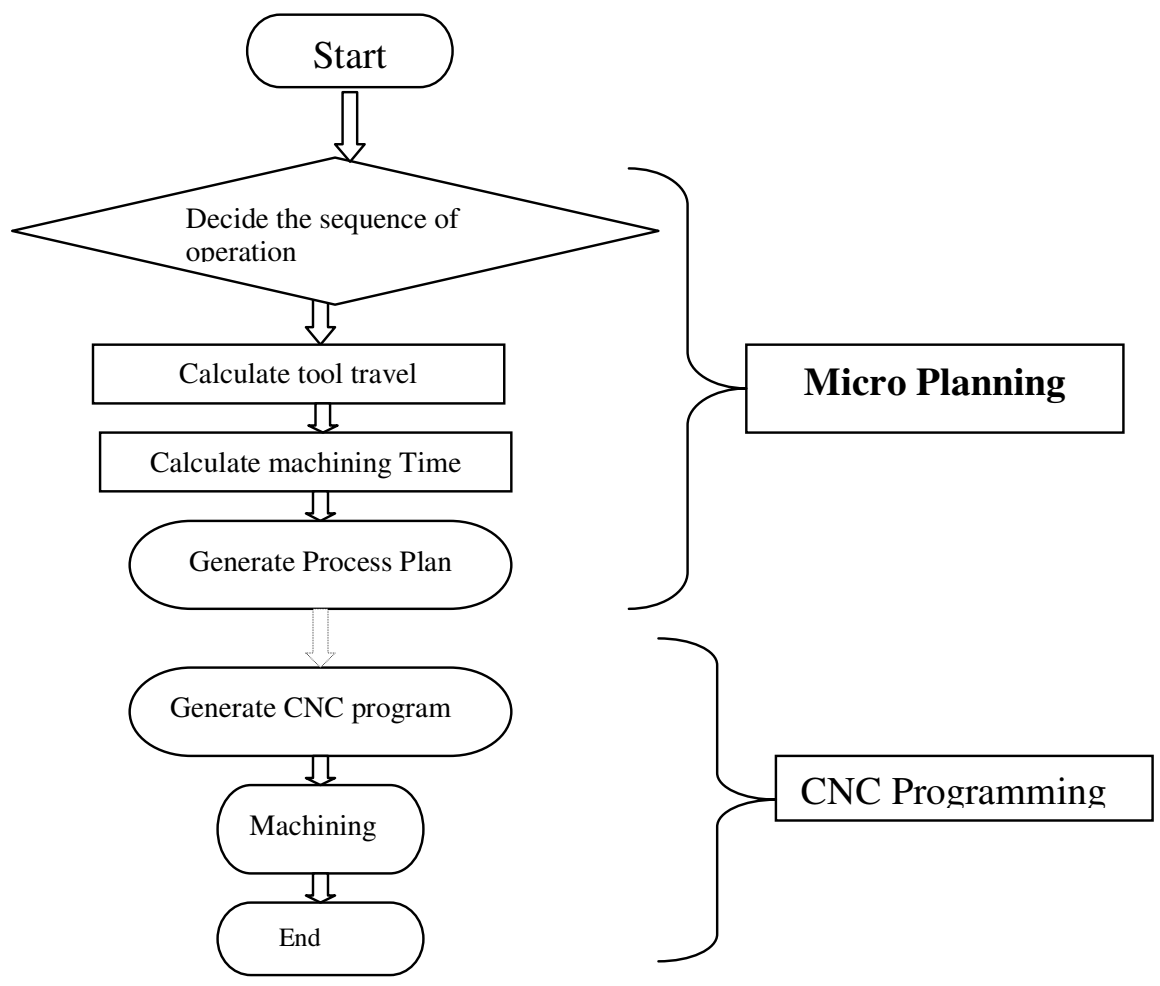

Fig.1 A structure of Micro planning and CNC programming

\section{DECISION RULES FOR SEQUENCING OF OPERATION}

1. The operation are selected according to feature having largest diameter first and then descending further in order of size from left to right, till smallest diameter is obtained.

2. Internal operations are considered only after external operations are completed.

3. The stock size is always taken greater than the finished size of workpiece.

4. The first operation in rotational component is always facing of extreme right face because face is considered as a reference for obtaining geometry of other features.

5. As the stock size selected is greater than the finished size of workpiece the second operation is always turning so as to obtain diameter of largest feature of a part on a cylindrical surface.

6. All plain turning (step turning) operations are completed first by converting every feature into a cylindrical surface.

7. All taper turning operation comes after step turning operation (sr.no.6). Taper turning operations are performed from right to left.

8. Finish cut operations of all cylinder, taper, recess and arc features are done before external threading, drilling, and internal threading. 
9. External threading operation comes in the sequence after completion of finish turning operation.

10. For internal operations first operation considered is a drilling and then boring.

11. If the hole size does not match with standard drill size, then boring operation is done after drilling.

12. Internal threading operation is performed only when required size of hole equal to major diameter is produced.

13. The last operation is always chamfering.

The built-in rules, as given above, are formulated on the basis of general machining principles and are used to define precedence relations among the features. The approach for operation selection is based on knowledge, the possible operation for each feature is determined heuristically. The program starts with selecting the features as per designed rule and then selecting possible operations for that feature. For example the hole feature can be done by two possibilities i.e. drilling, finish boring or drilling, rough boring, and finish boring. The 9 features and possible operation are described in the fig. 2 .

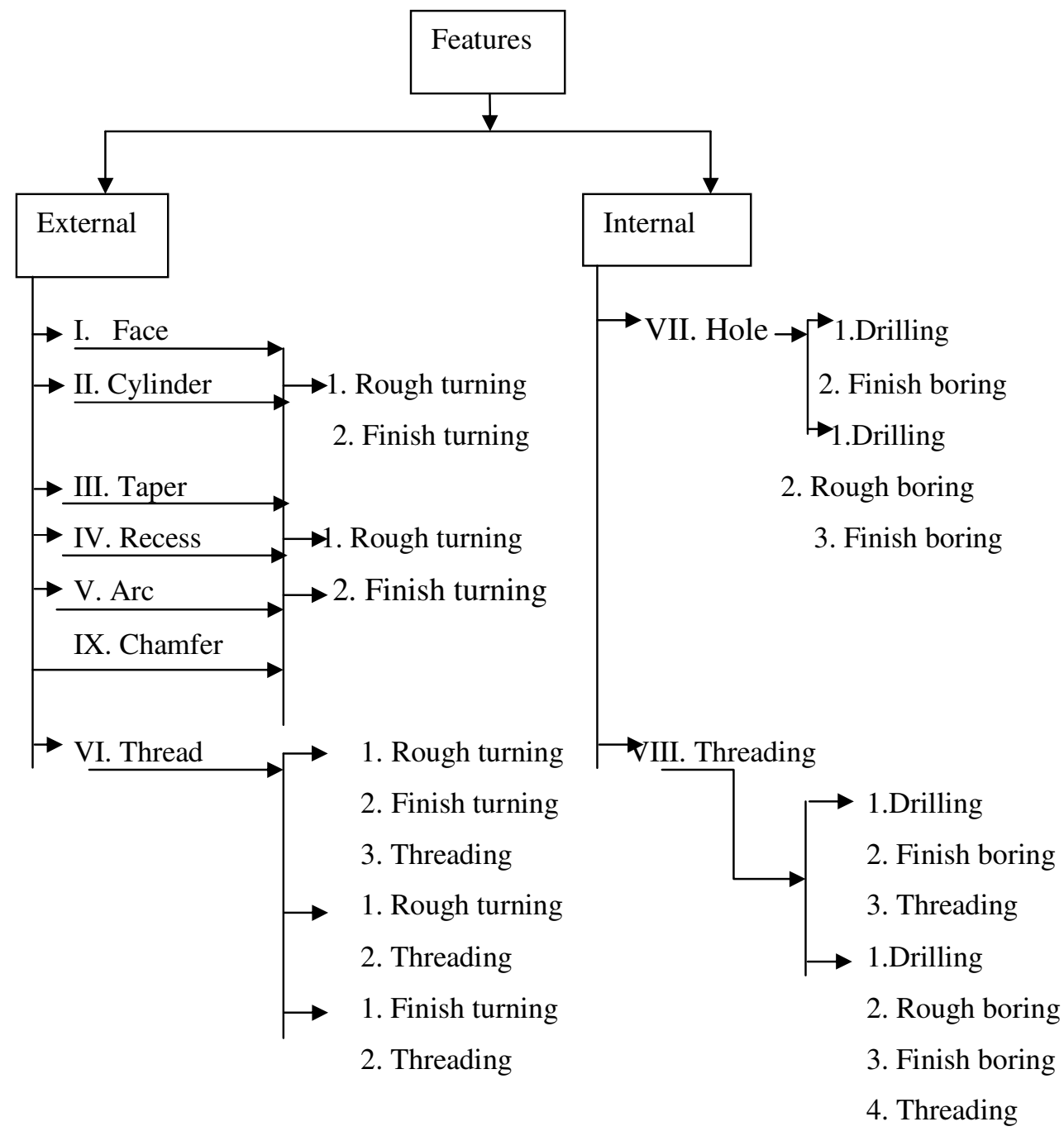

Fig.2 Feature-Operation Hierarchy 


\section{GENERATION OF CNC CODES}

The process plan developed for the given component using as input. The process plan is then used for generation of $\mathrm{CNC}$ codes. However, the process plan file is required to be imported again for identification of operation, tool travel and cutting parameters. It may be remembered here that if the operating system used for generation of process plan is different to the one used for part programming, the process plan file can not be imported. Under the circumstances, interfacing is required to make import process platform independent. Therefore a separate program is developed in VB6 for importing the process plan file.

\section{Case Study}

Part drawing is checked for concentricity of circle, redundant line, overlapping line and point in case of any corrections, the algorithm provides facility for revising the drawing accordingly. As shown in fig. 3 the part name is extracted from the file name of drawing which is given to the drawing in Auto CAD. The part name is displayed on the task bar of window as "Head pin" in the selected case study. The layers used in this case study for dimensions only. The remaining part drawing is displayed on "0" layer which occurs by default in Auto CAD. The part material is extracted from "material layer" and displayed on process sheet.

Fig.3 Part drawing

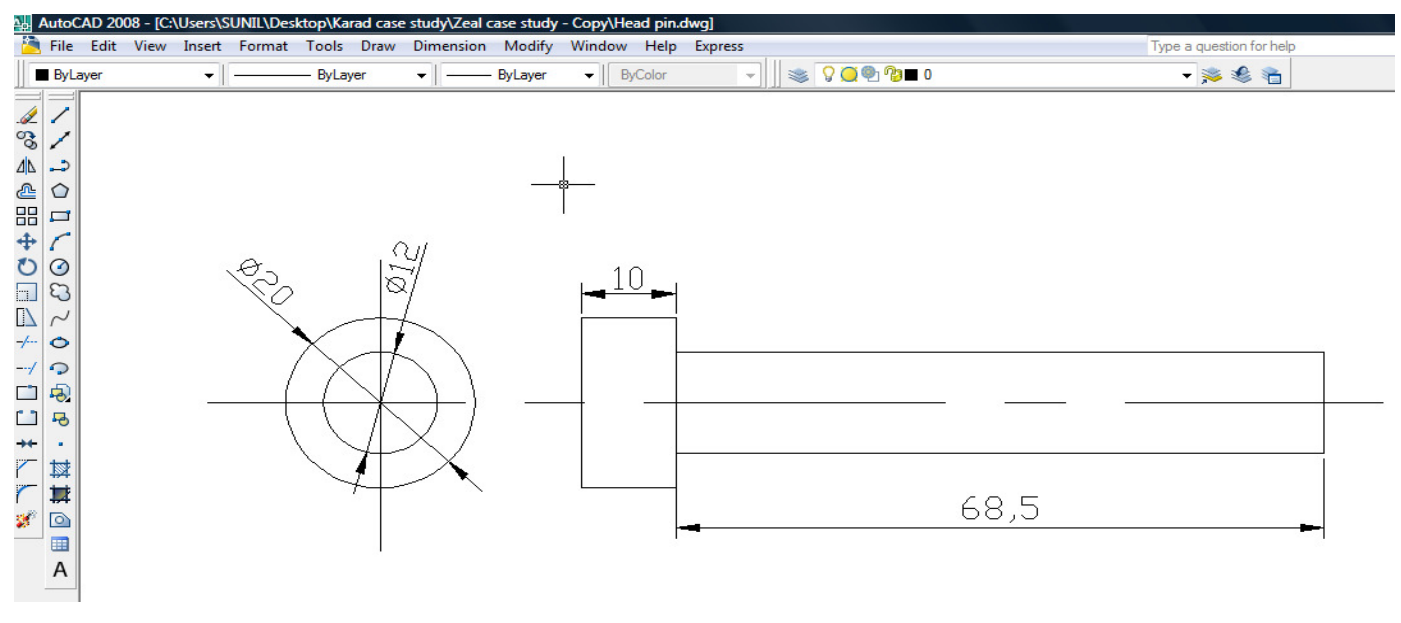

\subsection{Micro planning}

Next module on microplanning decides sequence of operations. The sequence of operation is decided on the basis of rules. It also includes number of repetitive operation (passes) when the material to be removed (depth) exceeds the allowable depth of cut. The stock size selected from data base is $22 \mathrm{~mm}$, which is nearer to part maximum diameter but more than the part max. diameter. For example, in case of step turning (second cylinder) the diameter is to be reduced from $21 \mathrm{~mm}$ to $13 \mathrm{~mm}$ which can not be done in one pass as allowable depth of cut is $1.5 \mathrm{~mm}$. Hence three passes of depth of cut $1.5 \mathrm{~mm}, 1.5 \mathrm{~mm}$ and $1 \mathrm{~mm}$ respectively are required. The finishing operation depth of cut is $0.5 \mathrm{~mm}$ is taken at the end of all plain turning operations. As per the procedure the sequence of operation starts and ends with facing operation of both sides. After incorporating the sequence of operation, a process plan is generated which includes description of cutting tool, cutting length and diameter, cutting parameters and machining time calculations. The process plan for "Head pin" is shown in fig.4. 


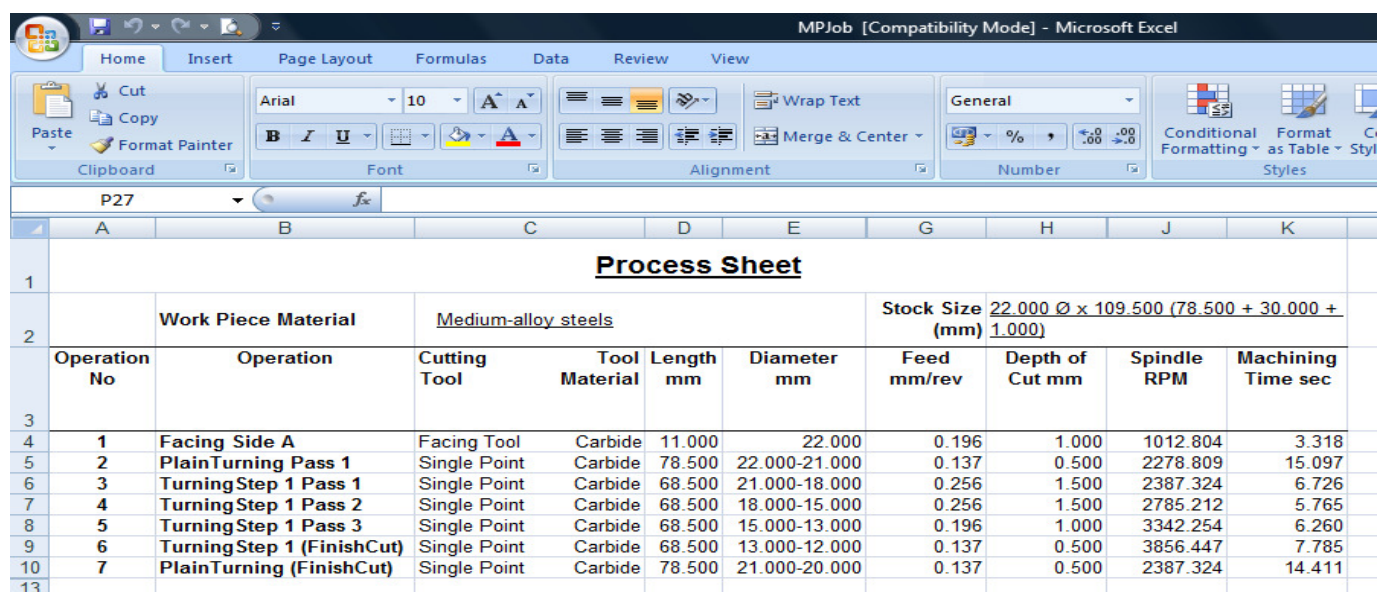

Fig.4 Process plan for "Head pin"

\subsection{CNC program}

N0010 G71G90G95M03M08 EOB

N0020 T01F0.20S1012.80 EOB

N0030 G00X13.00Z1.00 EOB

N0040 G00X0.00 EOB

N0050 G01Z-2.00 EOB

N0060 G01X-13.00 EOB

N0070 G00X13.00Z2.00 EOB

N0080 G01X-2.50T02F0.14S2278.81 EOB

N0090 G01Z-79.50 EOB

N0100 G00X2.50Z79.50 EOB

N0110 G01X-4.00T02F0.26S2387.32 EOB

N0120 G01Z-69.50 EOB

N0130 G00X4.00Z69.50 EOB

N0140 G01X-5.50T02F0.26S2785.21 EOB

N0150 G01Z-69.50 EOB

N0160 G00X5.50Z69.50 EOB

N0170 G01X-6.50T02F0.20S3342.25 EOB

N0180 G01Z-69.50 EOB

N0190 G00X6.50Z69.50 EOB

N0200 G01X-7.00T02F0.14S3856.45 EOB

N0210 G01Z-69.50 EOB

N0220 G00X7.00Z69.50 EOB 
N0230 G01X-3.00T02F0.14S2387.32 EOB

N0240 G01Z-79.50 EOB

N0250 G00X3.00Z79.50 EOB

N0260 M05 EOB

\section{CONCLUSiOnS}

In microplanning knowledge based approach is widely used. It is so because it incorporates all human skills, large amount of qualitative knowledge, and search procedure required to take various decisions in the form of decisions rules. The consistency in the decision can be maintained due to same decision rules used irrespective of complications in the part geometry. Moreover database approach is found more appropriate. It uses standard data relevant to specific machine and particular operation.

Manufacturing industries are combining material processing, material handling and part positioning system in to a single integrated manufacturing cell to offer flexibility, better quality and improved productivity. Process planning and part programming time directly affect the economic lot size. Automated process planning system can significantly enhance the throughput of such integrated cells and lower the economic lot size dramatically.

\section{REFERENCES}

[1] Kenneth Castelino, (2004), "AMPS - An automated modular process planning system" ASME, Journal of computing and information science in engineering, vol.04, pp235-241.

[2] Vijay Arora (1997) fixcapp “A fixture integrated computer aided process planning methodology. 17th AIMTDR conference 9-11 January 1997 R.E.C. Warangal, pp149-154.

[3] HMT "Production Technology"Revised edition, pp34,143.

[4] Zhenkai Liu (2007) "Sequencing of interacting prismatic machining features for process planning" Computers in Industry-58 pp295-303.

[5] Ali Oral (2004) "Automated cutting tool selection and cutting tool sequence Optimization for rotational part" Robotics and Computer- Integrated Manufacturing” Robotics and computer integrated manufacturing 20 , pp127-141.

[6] Mojtaba Salehi (2009) "Application of genetic algorithm to computer-aided process planning in preliminary and detailed planning" Engineering Applications of artificial Intelligence 22 pp11791187.

[7] Yuan-Jye Tseng(1999) “A modular modeling approach by integrating feature recognition and featurebased design" Computers in Industry-39 pp113-125.

[8] Yuguang $\mathrm{Wu}$ (2008) “Automated modular fixture planning based on linkage mechanism theory"Robotics and computer integrated manufacturing 24 , pp38-49.

[9] John M. Usher (2009) "Object Oriented approach to feature-based process planning", Design and implementation of intelligent manufacturing systems, pp275-300.

[10] Jian (John) Dong (2009) "Intelligent feature extraction for concurrent design and manufacturing" Design and implementation of intelligent manufacturing systems, pp301-323.

[11] Zhang Wei Bo (2006) “Optimization of process route by Genetic Algorithms" Robotics and computer integrated manufacturing 22, pp180-188. 